\title{
Evaluation of Porosity and Water Sorption in Conventionally Cured Modified Polymethyl Methacrylate Resin - An In Vitro Study
}

\author{
Merin Mathew ${ }^{1}$, Kamalakanth Shenoy ${ }^{2}$, Ravishankar K. S. ${ }^{3}$ \\ 1Department of Prosthetic Dental Sciences, Dental Materials, Jouf University, Sakaka, \\ Saudi Arabia. ${ }^{2}$ Department of Prosthodontics \& Dentistry, Dental Clinic, City plaza, \\ Mangalore, Karnataka, India. ${ }^{3}$ Department of Metallurgical \& Materials Engineering, \\ National Institute of Technology, Surathkal, Karnataka, India
}

\section{ABSTRACT}

\section{BACKGROUND}

Dimensional change and porosity in the polymethylmethacrylate based prosthesis affects its clinical performance. Hence, the present study aimed to evaluate the porosity and water sorption present in the modified polymethyl methacrylate polymer composite.

\section{METHODS}

Control group without fibre reinforcement and test groups with fibre reinforcement were prepared for the study. Three different fibres such as boron free-E glass fibre, untreated and plasma-treated polypropylene fibres in varying weight percentage and aspect ratio were considered for reinforcement. The porosity of the fractured surface was observed through a scanning electron microscope (scanning electron microscope) and sorption measured based on international standards organization (ISO) 1567:1999.

\section{RESULTS}

Control group exhibited porous structures, whereas all fibre-reinforced groups did not exhibit porous structure at the fracture surface. There was a significant difference in the sorption rate between control and test group $(p<0.001)$. Among fibrereinforced test groups, boron free E glass fibre reinforced polymethylmethacrylate exhibited maximum sorption followed by polypropylene fibre reinforced polymer test groups $(p<0.001)$. However, all samples showed sorption rate within the ISO specification.

\section{CONCLUSIONS}

Fiber reinforcement is an effective method to reduce porosity and water sorption in polymethylmethacrylate based polymer composite regardless of the fibre type.

\section{KEY WORDS}

Polymer Composite, Porosity, Water Sorption, Fiber Reinforcement,

\author{
Corresponding Author: \\ Dr. Merin Mathew, \\ Department of Prosthetic Dental Sciences, \\ Dental Materials, \\ Jouf University, \\ Sakaka, Saudi Arabia. \\ E-mail: dr.merin.mathew@jodent.org
}

DOI: $10.14260 / \mathrm{jemds} / 2021 / 201$

How to Cite This Article:

Mathew M, Shenoy K, Ravishankar KS Evaluation of porosity and water sorption in conventionally cured modified polymethyl methacrylate resin - an in vitro study. J Evolution Med Dent Sci 2021;10(13):930-934, DOI: 10.14260/jemds/2021/201

Submission 06-08-2020,

Peer Review 30-12-2020,

Acceptance 07-01-2021,

Published 29-03-2021.

Copyright (C) 2021 Merin Mathew et al. This is an open access article distributed under Creative Commons Attribution License [Attribution 4.0 International (CC BY 4.0)] 


\section{BACKGROUND}

The primary objective of prosthetic rehabilitation is to maintain oral health with appropriate function and aesthetics. Although implant therapy has established as a successful solution for edentulism, the conventional complete dentures remain popular among the majority of patients, primarily because of the economic limitations. ${ }^{1}$ One of the most widely used materials in prosthetic dentistry is polymethylmethacrylate because of its desirable characteristics like biocompatibility, chemical inertness, stability and ease in processing. It is the choice of material in dentistry for various rehabilitation procedures. ${ }^{2}$ It exhibits several advantageous properties as a dental restorative material. However, conventional polymethylmethacrylate is far from being ideal for denture base fabrication due to inferior mechanical and physical properties. ${ }^{1-3}$ Sorption and solubility of the polymethylmethacrylate material are one of the major concerns as the prosthesis needs to withstand the fluctuating $\mathrm{pH}$, temperature and masticatory forces in the oral environment. ${ }^{4}$ Water molecules exhibit affinity towards polymethylmethacrylate based materials and interact with the polymer chain and weaken the bonding between the macromolecules by separating them apart. This causes dimensional change and inferior mechanical properties of the denture base. ${ }^{5}$ Slight expansion due to water adsorption is considered as a beneficial characteristic to compensate for the processing shrinkage and to provide better fit of the denture bases in the mouth. ${ }^{2}$

Polymethyl methacrylate denture base material exhibits a lower rate of solubility. However, the presence of unreacted monomer in the processed denture can cause dissolution after fabrication, discolouration and low wear resistance of the material.6-8 Also, internal porosities created due to the release of residual monomer act as crack initiators and eventually lead to the fracture of the prosthesis in service during loading. ${ }^{9}$ The porosity in acrylic denture base resin remained to be a longstanding problem and nearly $11 \%$ porosity observed in it is a complex phenomenon that weakens the acrylic resin prosthesis due to accumulation of internal stresses. It leads to distortion, warpage and discolouration of the acrylic denture base. The volatilization of the monomer creates gaseous porosity and polymerization shrinkage contraction porosity. 4,6

Numerous studies were carried out on this material to bypass these complications and to enhance its features. Many studies suggest the modification of polymethylmethacrylate by preparing polymer blends and polymer composites. Polymer composites prepared with particulate filler and fibres. These types of materials exhibit properties that could not be achieved by any of the constituents alone, but combined properties of matrix material and the reinforced material. Various metallic and inorganic fillers and fibres are used for the preparation of polymethylmethacrylate composites. Different fibres such as nylon, carbon, aramid, polyethylene, polyester and glass fibres became popular to achieve desirable characteristics. $^{3}$ Some of the weak polymer matrix are replaced by a dispersed phase with lower water solubility and sorption characteristics. 10

However, the adhesion between fibre and polymer matrix is crucial as weak bonding between fibre and polymer matrix during polymerization may generate internal flaws and the fracture resistance thus compromised. Surface treated fibres combined with the polymer matrix enhance the bonding. ${ }^{11}$ The present study evaluated the porosity and solubility rate of three different types of modified polymethyl methacrylate material.

\section{METHODS}

\section{Materials}

Heat-activated polymethyl methacrylate denture base materials in the powder-liquid form obtained from dental products of India (DPI). Silane treated E-glass fibres from Goa glass fibres Ltd. Pure polypropylene fibre from Walter Enterprises, Mumbai, India. Type II, III gypsum products and modelling wax supplied by Hindustan Pvt. Ltd. Could mould seal separating medium purchased from DPI.

\section{Sample Size}

The comparison means formula used to calculate the sample size as 6 in each group $(n=168)$. The level of significance $Z \alpha$ and statistical power $\mathrm{Z} \beta$ considered $95 \%$ and $80 \%$ respectively. The difference among study groups (10) and the standard deviation (SD) chose from the pilot study (0.09)

\section{Characterisation of the Fibers}

The characterisation of fibres performed using scanning electron microscope, JOEL analytical, scanning electron microscope JSM 6380 LA. The surface of the fibres covered with a thin conducting film to allow scanning by a beam of electrons. The reflected electrons collected to implement the scanning on a cathode ray tube. The fibre surface analysed under various magnifications. ${ }^{12}$

\section{Characterisation of Boron Free-E Glass Fiber}

The purchased boron free-E glass fibre was solid, white to pale yellowish and no characteristic odour. The softening point was $850^{\circ} \mathrm{C}$ and density $2.6 \mathrm{~g} / \mathrm{cm}^{3}$. The diameter and the composition of the fibre identified using scanning electron microscope as shown in Figure 1

\section{Characterisation of Polypropylene Fiber}

The diameter of polypropylene fibre yarn measured using scanning electron microscope as in Figure 2 . The melting range $160-175^{\circ} \mathrm{C}$ and density $0.91 \mathrm{~g} / \mathrm{cm}^{3}$ noted.

\section{Plasma Treatment on Polypropylene Fiber}

Polypropylene fibre yarns vacuum treated in the plasma reactor for one hour. Plasma treatment on the fibre surface carried out for 10 minutes by hydrogen as a plasma carrier gas. The pressure inside the reactor maintained at 1.5 torr's with $540 \mathrm{eV}$ power. ${ }^{12}$ The plasma-treated surface observed under scanning electron microscope to understand the difference in the surface texture of untreated and plasma-treated fibre surfaces (Figure 3)

\section{Preparation of the Mould}

Modelling wax cut into the desired dimension and invested in the dental flask using type II \& III gypsum products. Dewaxing 
performed after an hour and the obtained mould cleaned using soap solution. A thin layer of cold mould seal applied as separating the medium and allowed to dry. ${ }^{12}$

\section{Preparation of the Samples}

Polymer and monomer, $2.4 \mathrm{gm}: 1 \mathrm{ml}$ mixed in a porcelain jar and allowed to reach dough consistency for the preparation of the control group. For the reinforced groups, different fibres, considered $\mathrm{A}=$ Boron free $\mathrm{E}$ glass fibre $20 \mu \mathrm{m}$ diameter, $\mathrm{B}=$ Untreated and C = Plasma-treated polypropylene fibre $220 \mu \mathrm{m}$ diameter of varying lengths ( $3 \mathrm{~mm}, 6 \mathrm{~mm}, 12 \mathrm{~mm})$ and weight, percentages ( $2.5 \mathrm{Wt} \%, 5 \mathrm{Wt} \%, 10 \mathrm{Wt} \%$ ) impregnated in the monomer liquid for 5 minutes. The polymer powder then mixed and allowed to reach the dough consistency. The dough kneaded and packed into the mould and the closed flask kept in a hydraulic press apparatus for bench curing under 1400 Psi for 30 minutes. Flask with clamp then transferred into the water bath in the acryliser. The temperature then elevated to $72^{\circ} \mathrm{C}$ and maintained for 90 minutes, followed by $100^{\circ} \mathrm{C}$ for 60 minutes to achieve maximum polymerisation of the samples. The flask is then permitted to cool in the same water bath to room temperature and the acrylic resin specimens retrieved after deflasking. Obtained specimens cleaned from stone particles and polished using sandpaper 80, 120 and 150 grits. Each sample visually inspected calibrated, polished using pumice. ${ }^{12}$

\section{Microstructure and Porosity Estimation}

The presence of porosity is observed under scanning electron microscope at the fractured surfaces for all samples prepared in the present study. Characterisation of the samples carried out using scanning electron microscope at different accelerating voltages of $5 \mathrm{kV}$ and $10 \mathrm{kV}$.

\section{Dissolution / Water Sorption}

Dissolution or sorption rate of the samples evaluated based on international standards organization (ISO) 1567:1999 by evaluating the weight shift of the samples after thirty days of immersion in the distilled water. Initial weight measured and noted as W1. The samples then kept in distilled water for thirty days. The water bath maintained at $37^{\circ} \mathrm{C}$. Samples then retrieved after immersion and dried in a vacuum oven at $37^{\circ} \mathrm{C}$ and weighed as W2. The weight difference identified as W3 (W2 - W1).

\section{Statistical Analysis}

The results analysed using one-way analysis of variance, followed by Tukey Kramer multiple comparison test using GraphPad InStat software. Null hypothesis HO set as there is no effect in the porosity and dimension as a result of fibre incorporation in the polymethyl methacrylate polymer.

\section{RESULTS}

Porosity Observed in Control and Test Samples The scanning electron microscope analysis of fracture surface exhibited porous structure in control samples (Figure 4a).
Porosities can be observed at the fractured surface of the polymethylmethacrylate without any fiber reinforcement in it. No porous structure recognized in the fibre-reinforced test groups (Figure 4b-d). Only fibre pull out observed in all three fibre reinforced samples. Large void present in the figure $4 \mathrm{c}$ represents the fibre pull out. In figure $4 \mathrm{~b}$ and $4 \mathrm{~d}$ fibre and matrix fracture only observed without any porous areas.

\section{Dissolution / Sorption of Control Sample}

All tested samples did not cause significant dissolution or sorption during immersion. A weight increase of $14.83 \mu \mathrm{g} /$ $\mathrm{mm}^{3}$ observed for control samples after immersion in the distilled water. However, the results obtained are less than the maximum value (32 $\mathrm{\mu g} / \mathrm{mm}^{3}$ ) recommended by ISO 1567:1999. The fibre reinforced test groups exhibited less significant gain in the weight compared to the control group after immersion (Table 1).

Among the fibre reinforced test groups, maximum weight gain identified in the boron free-E glass fibre reinforced group followed by the untreated polypropylene fibre reinforced groups and no weight change observed with the plasmatreated fibre reinforced test group (Figure 5).

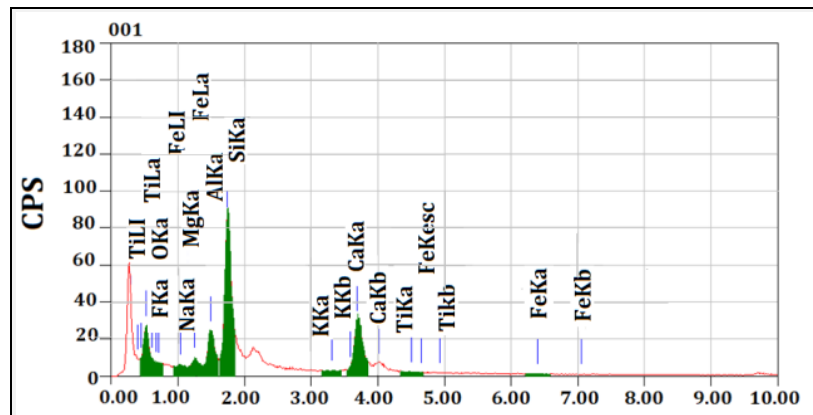

Figure 1. Characterization (scanning electron microscope-EDX) of Boron Free E Glass Fiber
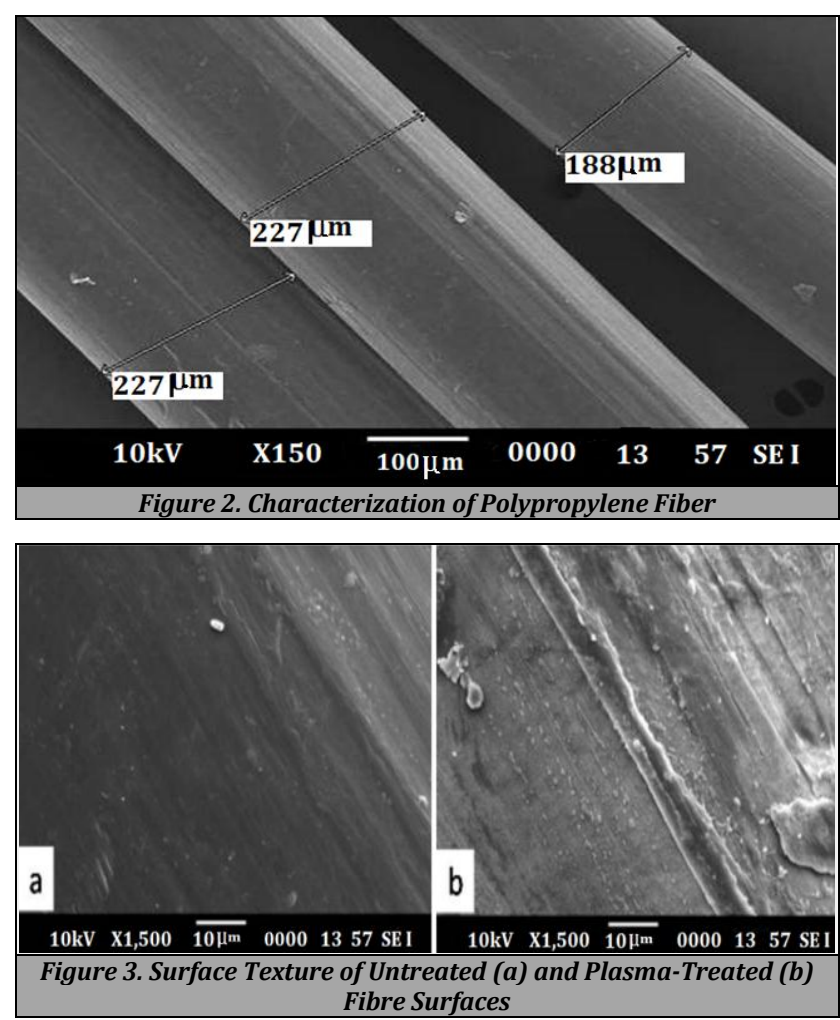

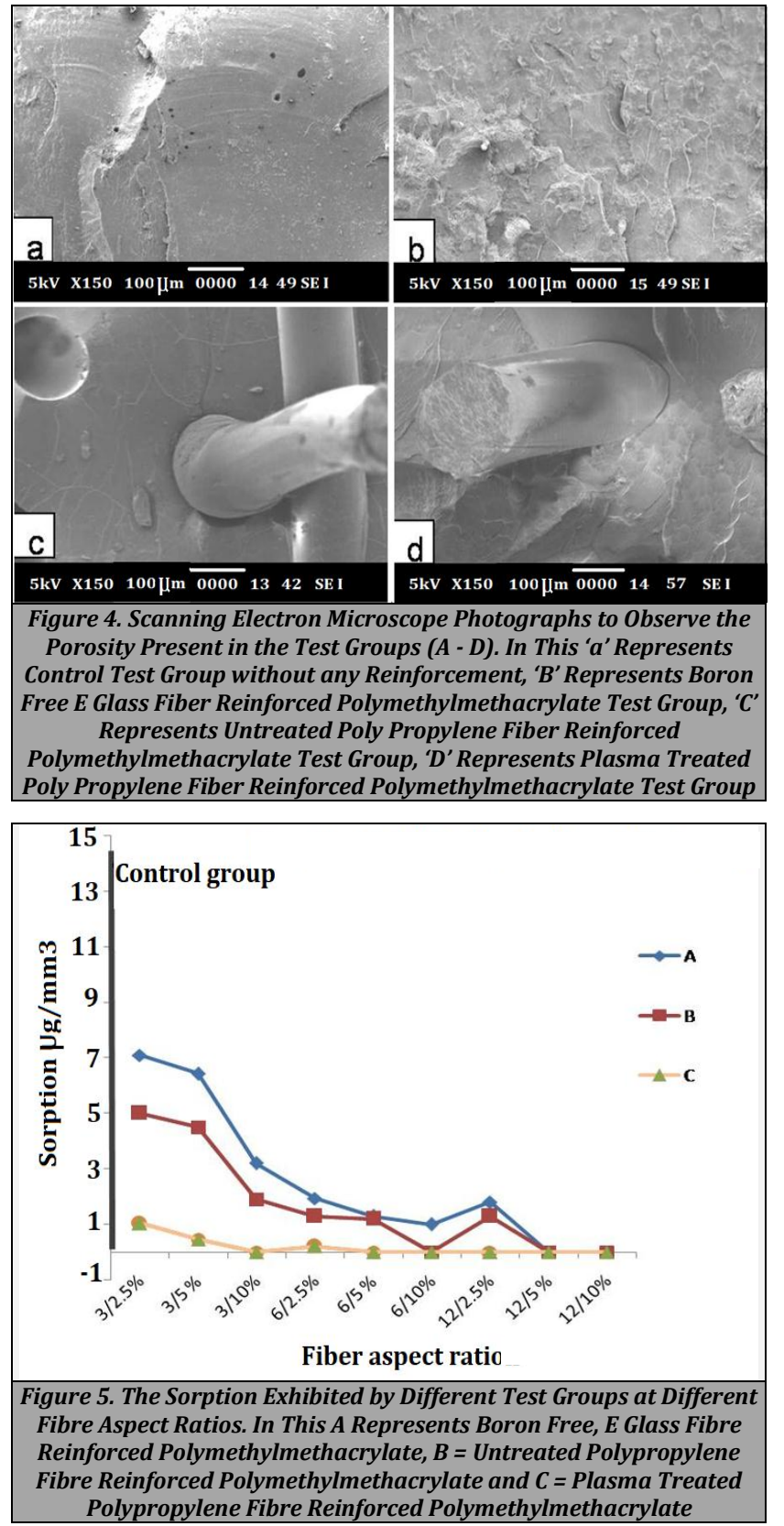

\begin{tabular}{|cccc|}
\hline $\begin{array}{c}\text { Control } \\
\text { Sample }\end{array}$ & \multicolumn{3}{c|}{$14.83 \pm 0.0004$} \\
& & &
\end{tabular}

\section{DISCUSSION}

Researchers suggest that compression moulding technique reduces the occurrence of porosity as a result of even distribution of compressive force during polymerization. ${ }^{13-14}$ Therefore the present study utilized conventional heat curing compression moulding technique for the fabrication of samples for porosity and solubility measurement. Some porous structures were observed in control samples whereas the fractured area of polymer reinforced test groups did not show porosity, which indicates that the presence of fibres adhered to the matrix lessens the chance for porous structure rather than using the polymer matrix alone. ${ }^{15}$

In the present study, the samples were immersed in distilled water to observe the stability of prepared polymer composite. Lung and Darvel reported that water sorption or solubility results in the acrylic prosthesis when kept in an aqueous medium, and it occurs mainly by leaching out the unreacted monomer and water-soluble additives into oral fluids. Zero percentage of the unreacted monomer is unrealistic and hard to achieve in practice. ${ }^{6}$

The weight gained in the control test samples after 30 days immersion in distilled water is considered as an advantage. Harrison et al. reported that water adsorbed into the surface of the polymethylmethacrylate denture base alter its dimension slightly but it results in the better fit and retention of the prosthesis by intimate contact with underlying edentulous ridge. ${ }^{16}$ Water molecules are absorbed into the polymethylmethacrylate based material due to its polar nature. ${ }^{10}$ This causes slight mobility of the macromolecules which leads to expansion of the prosthesis, and thereby relaxation of stress occurring during heat curing. ${ }^{10,16-17}$ The results obtained in the present study observed that the weight loss due to immersion was nil and this might be due to the fewer residual monomer because the unreacted monomer or other additives present in the fabricated prosthesis lead to dissolution. ${ }^{18-21}$

Table 1 represents the weight changes occurred in the control and test groups. There has been a significant difference in weight change between control and test groups $(\mathrm{p}<0.001)$. Even though the sorption is lower than the maximum value recommended by ISO, weight gain noted as in different research reports. ${ }^{19-20}$ Increased rate of polymerization reaction might have occurred in silane treated E-glass fibres incorporated in polymethylmethacrylate as a result of silane treatment ${ }^{22}$ and compression moulding. ${ }^{14}$ Polypropylene fibre exhibits a lower rate of water sorption. Thus no dimensional change expected with humidity change, ${ }^{23}$ this reduces the chances of absorbing the water by fibres in the polypropylene fibre reinforced test groups. However, due to the adsorption nature of the polymethyl methacrylate resin, and the lack of fibre matrix interaction and adhesion, little but negligible dimensional changes are observed for the untreated polypropylene fibre reinforced test groups $(\mathrm{p}<0.001)$.

Plasma-treated fibre reinforced test group exhibited minimum weight change. The plasma treatment enhances the surface energy of fibres. Thereby fibre-matrix interaction increases, and the number of residual monomers decreases. ${ }^{24}$ 25 The polymethylmethacrylate matrix material is vulnerable to sorption when it is immersed in an aqueous medium, ${ }^{4}$ but a complete polymerization reaction reduces the chances of dissolution or sorption. ${ }^{6}$ 
As depicted in figure 5, irrespective of the fibre incorporated, a similar trend exhibited in all test groups with the aspect ratio. Within the limit, increased fibre content decreased the dimensional change. Therefore, the null hypothesis is rejected.

\section{CONCLUSIONS}

Fibre reinforcement reduces the chances of occurrence of porosity in the polymer composite regardless of fibre type. Among fibre reinforced groups, boron free-E glass fibre reinforced polymethylmethacrylate groups exhibited higher dimensional change compared to polypropylene fibre reinforced polymethylmethacrylate groups. Plasma treatment on polypropylene fibre significantly reduced the water sorption of polymer composite prepared.

Data sharing statement provided by the authors is available with the full text of this article at jemds.com.

Financial or other competing interests: None.

Disclosure forms provided by the authors are available with the full text of this article at jemds.com.

\section{REFERENCES}

[1] Frazer RQ, Byron RT, Osborne PB, et al. polymethylmethacrylate: an essential material in medicine and dentistry. J Long Term Eff Med Implants 2005;15(6):629-39.

[2] Ajay R, Suma K, Ali SA. Monomer modifications of denture base acrylic resin: a systemic review and meta analysis J Pharm Bioallied Sci 2019;11(Suppl 2):S112-25.

[3] Gad MM, Fouda SM, Al-Harbi SA, et al polymethylmethacrylate denture base material enhancement: a review of fiber, filler and nanofiller addition. Int J Nanomedicine 2017;12:3801-12.

[4] Figueroa RMS, Conterno B, Arrais CAG, et al. Porosity, water sorption and solubility of denture base acrylic resins polymerized conventionally or in microwave. J Appl Oral Sci 2018;26:e20170383.

[5] Savirmath A, Mishra V. A Comparative evaluation of the linear dimensional changes of two different commercially available heat cure acrylic resins during three different cooling regimens. J Clin Diagn Res 2016;10(11):ZC50-4.

[6] Lung CYK, Darvell BW. Minimization of the inevitable residual monomer in denture base acrylic. Dental Mater 2005;21(12):1119-28.

[7] Bacali C, Baldea I, Moldovan M, et al. Flexural strength, biocompatibility, and antimicrobial activity of a polymethyl methacrylate denture resin enhanced with graphene and silver nanoparticles. Clin Oral Invest 2020;24(8):2713-25.

[8] Alamgir M, Mallick A, Nayak GC, et al. Development of polymethylmethacrylate/ $\mathrm{TiO}_{2}$ nanocomposites as excellent dental materials. Journal of Mechanical Science and Technology 2019;33(10):4755-60.

[9] Badr NA, Ibrahim AM, Mohamed GF. Effect of the curing mode on the clinical performance and properties of acrylic resin overdenture bases. Cairo Dental Journal 2008;24(2):259-71.

[10] AL-Omari AW, Kassab NH, Mohammed NZ. Water sorption and solubility of two acrylic resin denture base materials polymerized by infrared radiation. Al-Rafidain Dent J 2019;19(1):20-31.

[11] Zindani D, Kumar K. An insight into additive manufacturing of fiber reinforced polymer composite. International Journal of Lightweight Materials and Manufacture 2019;2(4):267-8.

[12] Mathew M, Shenoy K, Ravishankar KS. Flexural strength of hydrogen plasma-treated polypropylene fiber-reinforced polymethyl methacrylate denture base material. J Indian Prosthodont Soc 2018;18(3):257-62.

[13] Rajak DK, Pagar DD, Kumar R, et al. Recent progress of reinforcement materials: a comprehensive overview of composite materials. J Mater Res Technol 2019;8(6):6354-74.

[14] Gharechahi J, Asadzadeh N, Shahabian F, et al. Effect of molding technique on two physical properties of acrylic resin specimens. N Y State Dent J 2016;82(4):38-44.

[15] Shesan OJ, Stephen AC, Chioma AG, et al. Fiber-matrix relationship for composites preparation. Intechopen 2019. DOI: 10.5772 /intechopen.84753.

[16] Harrison A, Huggett R, Zissis A. Measurement of dimensional accuracy using linear and scanning profile technique. Int J Prosthodont 1992;5(1):68-72.

[17] Das G, Khokhar M, Naeem S, et al. Comparison of solubility and water sorption of two different soft lining material. J Ayub Med Coll Abbottabad 2018;30(2):175-9.

[18] Palaskar JN, Singh S, Mittal S. Evaluation and comparison of different polymerization techniques, curing cycles, and thicknesses of two denture base materials. Indian J Dent Res 2019;30(4):583-9.

[19] Chai J, Takahashi Y, Hisama K, et al. Water sorption and dimensional stability of three glass fiber-reinforced composites. Int J Prosthodont 2004;17(2):195-9.

[20] Zirak M, Vojdani M, Mohammadi S, et al. Comparison of the water sorption and solubility of four reline acrylic resins after immersion in food-simulating agents. J Int Soc Prev Community Dent 2019;9(1):40-6.

[21] Tham WL, Chow WS, Mohd Ishak ZA. Simulated body fluid and water absorption effects on poly (methyl methacrylate)/hydroxylapatite denture base composites. Express polymer letters 2010;4(9):517-28.

[22] Larson TD. The uses of silane and surface treatment in bonding. Northwest Dent 2006;85:27-30.

[23] Deepan N, Prakash AC, Rao KB, et al. In Vitro evaluation and comparison of transverse and impact strength of heat polymerized acrylic resin reinforced with polyethylene fibers and polypropylene fibers. Journal of Advanced Medical and Dental Sciences Research 2014;2(2):46-56.

[24] Chu PK, Chen JY, Wang L, et al. Plasma surface modification of biomaterials. Material Science and Engineering 2012;36(5):143-206.

[25] Slepicka P, Kasalkov NS, Stranská E, et al. Surface characterization of plasma treated polymers for applications as biocompatible carriers. express Polymer Letters 2013;7(6):535-45. 